J Health Econ. 2018 September ; 61: 244-258. doi:10.1016/j.jhealeco.2018.01.005.

\title{
The effect of integration of hospitals and post-acute care providers on Medicare payment and patient outcomes
}

\author{
R. Tamara Konetzka ${ }^{a}$, Elizabeth A. Stuart ${ }^{b}$, and Rachel M. Werner ${ }^{c, d},{ }^{*}$ \\ aDepartment of Public Health Sciences, University of Chicago, United States \\ bepartment of Mental Health, Johns Hopkins Bloomberg School of Public Health, United States \\ 'Division of General Internal Medicine, University of Pennsylvania, United States \\ ${ }^{d}$ Center for Health Equity Research and Promotion, Crescenz VA Medical Center, Philadelphia, \\ PA, United States
}

\section{Abstract}

In this paper we examine empirically the effect of integration on Medicare payment and rehospitalization. We use 2005-2013 data on Medicare beneficiaries receiving post-acute care (PAC) in the U.S. to examine integration between hospitals and the two most common post-acute care settings: skilled nursing facilities (SNFs) and home health agencies (HHA), using two measures of integration-formal vertical integration and informal integration representing preferential relationships between providers without formal relationships. Our identification strategy is twofold. First, we use longitudinal models with a fixed effect for each hospital-PAC pair in a market to test how changes in integration impact patient outcomes. Second, we use an instrumental variable approach to account for patient selection into integrated providers. We find that vertical integration between hospitals and SNFs increases Medicare payments and reduces rehospitalization rates. However, vertical integration between hospitals and HHAs has little effect, nor does informal integration between hospitals and either PAC setting.

\section{Keywords}

Post-acute care; Vertical integration; Medicare

\section{JEL classifications}

I11; I18

\section{Background}

Vertical integration has long been of interest to economists, but vertical integration in health care settings remains understudied. The scarcity of evidence has become more apparent as new policies are implemented that directly or indirectly encourage vertical integration in

This is an open access article under the CC BY-NC-ND license (http://creativecommons.org/licenses/by-nc-nd/4.0/).

*Corresponding author at: University of Pennsylvania, United States. rwerner@upenn.edu (R.M. Werner). 
order to increase coordination across settings. These policies are motivated by accumulating evidence from the medical and health services literature suggesting that poorly coordinated care is unsafe for patients and expensive for the health care system (Beers et al., 1992; Moore et al., 2003; Coleman and Berenson, 2004; Van Walraven et al., 2004; Coleman et al., 2005a,b).

Vertical integration was a common approach to attempt to improve coordination in the mid-1990s (Baicker and Levy, 2013). With the popularization of managed care, many tertiary-care hospitals acquired closely affiliated services that complement hospital services, such as smaller community-based hospitals, physician practices, and post-acute care providers (Cutler and Scott Morton, 2013). By the end of the 1990s there had been significant market consolidation, with the number of acute-care hospitals declining by $15 \%$ (Cutler and Scott Morton, 2013) and three-quarters of the remaining hospitals having acquired a supplier of post-acute care (Congressional Budget Office, 1997).

More recently, attention has again turned to integration in the presence of multiple emerging policies attempting to use financial incentives to improve care coordination. For example, under the Affordable Care Act, Medicare is experimenting with Accountable Care Organizations (ACOs) where payers and providers take on responsibility for managing the health of their members across health care settings and are financially accountable for providing high-quality and low-cost care (Centers for Medicare and Medicaid Services, 2013). Pay-for-Performance programs, now widespread across the health care spectrum, reward providers financially for meeting quality goals (Kondo et al., 2016). Some of the metrics used in pay-for-performance programs, such as reduced inpatient readmissions, implicitly encourage coordination across providers. Other examples of payment reforms that encourage coordination include bundled payments, which combine payments across provider settings for a single episode of care, and financial penalties for hospitals with higher-than-expected readmission rates (Centers for Medicare and Medicaid Services, 2013). Although payment bundling is still in the demonstration phase and evidence on ACOs and readmissions penalties is inconclusive, these policies are likely to create new incentives for providers to optimize care across health care settings, potentially through integration.

While integration and market consolidation is expected in response to these emerging policies, the effects of market consolidation may be at odds with the intended policy effects. The policies are designed to encourage coordination of care with the intended effect of improving quality and constraining Medicare spending. At the same time, the achievement of those goals likely depends on the underlying payment policy, the strength of the incentives, and the countervailing anti-competitive effects of consolidation.

Integration may be formal, defined by combined legal ownership, or informal, whereby organizations selectively form strong ties with other organizations through mechanisms such as shared electronic health records, sharing physicians or nurses across settings to promote continuity, or preferentially sharing patients, though remaining legally separate while doing so. The effects of integration may depend on the details of the relationship, but because these mechanisms generally require resources, they typically only make sense in situations when the volume of shared patients is large relative to other potential partners in the market. 
There is a large literature on the effects of horizontal integration. While horizontal mergers nearly always reduce competition, the effects of reduced competition on quality and costs are surprisingly mixed. In the hospital setting with regulated prices (i.e. Medicare payment) reduced competition nearly always reduces quality. With unregulated prices, the effects of reduced competition on quality and costs are uncertain (Gaynor and Town, 2012).

The effects of vertical integration on quality and spending are also ambiguous, both theoretically and empirically. Theoretically, vertical integration can serve to achieve efficiencies by facilitating coordination of care, addressing incomplete contracting problems, and otherwise reducing transaction costs (Robinson, 1996). On the other hand, vertical integration can enhance or extend market power by limiting rivals' access to the integrated firm or by raising rivals' costs by charging higher prices than the internal transfer price of the integrated firm. Vertical integration can also be inefficient without being anticompetitive, simply by reducing choice (Gaynor and Town, 2012).

Empirical studies examining the effects of vertical integration in health care are sparse. Two papers examined the effect of vertical integration on competition in the case of hospitalphysician practice integration in the 1990s (Ciliberto and Dranove, 2006; Cuellar and Gertler, 2006). These studies find conflicting results, with one finding that the anticompetitive effects of integration increased prices (Cuellar and Gertler, 2006) and the other finding no effect (Ciliberto and Dranove, 2006). Research on the effect of vertical integration on efficiency is similarly lacking, with studies on hospital-physician integration in the 1990s finding little relationship between integration and quality and costs (Madison, 2004; Burns and Muller, 2008). However, these studies use data that is close to two decades old, and most fail to account for the endogenous relationship between a hospital's decision to integrate and its quality and costs. Two more recent studies have examined the effect of hospital ownership of physician practices on health care prices, finding that vertical integration in the early 2000s was associated with higher prices and spending (Baker et al., 2014; Neprash et al., 2015). However, like earlier research, these studies failed to fully account for patient selection into integrated practices and the endogeneity between prices and integration.

Two prior papers have examined the effect of vertical integration on practice patterns in post-acute care using data on integration of hospitals with home health agencies and skilled nursing facilities. David et al. (2013) conceptualize vertical integration as a solution to an incomplete contracting problem, whereby integration allows tasks that are substitutable across settings to be more efficiently allocated to the lower-cost setting (the post-acute care or PAC setting). They find that hospitals that are vertically integrated tend to discharge patients to post-acute care quicker and sicker, yet health outcomes are the same or better, suggesting that vertical integration reduces coordination problems (David et al., 2013).

More recently, Rahman et al. (2016) examine differences in outcomes for patients going to hospitals that are vertically integrated with skilled nursing facilities (SNFs) vs hospitals that are not integrated. Using data from 2009, they find that patients discharged from a hospital to a vertically integrated SNF have shorter length of stay in the SNF (with associated lower Medicare spending on SNFs) and more days in the community. They find no difference in 
patient outcomes other than a slightly lower rate of readmissions during the first week of a SNF stay for patients going to a vertically integrated provider.

We build on these papers in several key ways. First, we pay careful attention to endogeneity in both patients' selection into vertically integrated post-acute care providers and in hospitals' decision about whether to vertically integrate. Rahman, Norton and Grabowski address the former (patient selection) by using an instrumental variables approach, but treat the hospitals' decision about whether to integrate as exogenous. David, Rawley, and Polsky, on the other hand, address the latter (endogeneity in the hospitals' decision) using instrumental variables, but do not directly address patient selection based on unobservable factors. We address both potential sources of endogeneity, using an instrumental variables approach for patient selection, and then use provider fixed effects to identify the effect of changes in vertical integration over time. Second, both prior studies use just one year of data (David, Rawley, and Polsky use 2006 while Rahman, Norton, and Grabowski use 2009). Thus, they were limited to a cross-sectional analysis. In contrast, we use a 2005-2013 panel of data, which enables a longitudinal analysis of organizations that changed integration status over time, and also may be more reflective of the type of integration. Third, we consider informal integration in addition to the more formal vertical integration, an understudied area.

Our contribution is, therefore, to provide a more complete and updated picture that takes advantage of longitudinal data to address endogeneity concerns, better measurement, and more recent data that are relevant to the current policy context. Specifically, we use Medicare claims data to examine the effects of integration (vertical and informal) between hospitals and PAC providers on Medicare spending and hospital readmissions. We find that hospitals that vertically integrate with SNFs are able to take advantage of the structure of the payment system to increase Medicare payments overall, mostly through increasing the number of SNF days, while reducing hospital readmissions. Little effect of formal vertical integration on either outcome is found for patients discharged to home health agencies (HHAs), likely due to differences in the payment system for this setting. We also find that informal integration has no effect on Medicare payments or read-missions.

\section{The post-acute care context}

One setting where the effects of uncoordinated care have received significant attention is care during the post-hospital-discharge period. One-fifth of Medicare beneficiaries are rehospitalized within 30 days of hospital discharge and more than one-third are rehospitalized within 90 days (Jencks et al., 2009). Some estimate that nearly $90 \%$ of these readmissions are unplanned and potentially preventable, translating into a $\$ 17$ billion expenditure for Medicare, or nearly 20\% of Medicare's acute-care payments (Centers for Medicare and Medicaid Services, 2007).

An expanding evidence base suggests that high readmission rates are related to serious deficits in the coordination of care across sites of care. Qualitative studies have demonstrated that when patients are discharged home, they are often unprepared for self-management and unable to reach a health care provider familiar with their care plan when questions arise 
(vom Eigen et al., 1999; Harrison and Verhoef, 2002). Quantitative studies document high rates of medication errors, incomplete or inaccurate information transfer, and lack of appropriate follow-up care for vulnerable patients during transitions, which compromise quality and patient safety (Beers et al., 1992; Van Walraven et al., 2002; Moore et al., 2003; Coleman et al., 2005b). Collectively, these problems in transitions and coordination increase rates of hospital readmission and health care costs.

Medicare pays for the vast majority of PAC, spending $\$ 62.1$ billion on PAC in 2012 (MedPAC, 2013a,b), with over 5 million Medicare beneficiaries using some form of PAC in 2011 (MedPAC, 2013a,b). Approximately 38\% of all Medicare discharges from hospitals are to PAC. The vast majority of PAC is provided by two key types of organizations: SNFs accounting for $49 \%$ of discharges to PAC and HHAs accounting for $43 \%$.

Medicare's costs of PAC have grown dramatically in recent decades. After Medicare implemented its hospital prospective payment system in the 1980s, hospitals responded by minimizing hospital length of stay, creating an increased demand for PAC and many new PAC providers. As PAC was primarily a fee-for-service sector at the time, the growing use of PAC was accompanied by little incentive for efficient use. Dramatic spending growth led to the implementation of prospective payment systems for PAC following the Balanced Budget Act of 1997. However, even under prospective payment, the use of PAC has continued to grow, in part due to increased concerns over patient safety and the recognized need to provide better transitions between inpatient and outpatient care. Between 1994 and 2009 Medicare spending on PAC was the fastest growing major spending category for Medicare, accounting for a large portion of Medicare's overall spending growth over that time period (Chandra et al., 2013).

In summary, Medicare's PAC sector is large, expensive, and growing in both spending and policy focus. Examining the effects of vertical integration on patient outcomes and Medicare spending in the setting of hospitals and PAC providers thus provides a unique opportunity to shed light on a fundamental question that is also of current policy importance.

\section{Conceptual framework}

We assume that both hospitals and PAC providers choose levels of inputs to maximize utility, which may derive from both profits and nonmonetary goals. More specifically, each provider (hospital and PAC) chooses the level of inputs $I$ of care in order to maximize the utility $U$ it gets from profits and patient outcomes $Q$ :

$$
\begin{gathered}
\max _{I_{h}} U_{h}\left[\pi_{h}\left(I_{h}\right), Q_{h}\left(I_{h}\right)\right] \\
\max _{I_{P A C}} U_{P A C}\left[\pi_{P A C}\left(I_{P A C}\right), Q_{P A C}\left(I_{P A C}\right)\right]
\end{gathered}
$$


In the absence of vertical integration, these utility functions remain separate. Optimal inputs are conditional on incentives inherent in the Medicare payment system, where both hospitals and PAC providers are paid under prospective payment systems (PPS). For hospitals under the Inpatient PPS (IPPS), each admission is categorized into a diagnostic-related group (DRG) that has a payment assigned to it based on expected resources use. This has created strong incentives for hospitals to keep patients no longer than medically necessary. Indeed, hospital length of stay has significantly shortened since the introduction of IPPS (Guterman and Dobson, 1985; Feder et al., 1987; Manton et al., 1993) and hospitals continue to focus on finding ways to decrease length of stay and inpatient costs within the bounds of medically appropriate care.

PAC is also paid prospectively, but the details of the payment systems vary in salient ways by setting. SNF payments are a per diem rate set by expected service intensity during the stay. The classification of expected service use is reassessed and potentially adjusted at regular intervals over time as the patient's condition changes, but the per diem payment rate within each interval is predetermined. In contrast, HHA payments are episodic with payments adjusted for illness severity and set for a 60-day episode regardless of number of expected visits. In both cases, limited ex post adjustments are made to payments in particular cases, such as lower payments to HHAs for particularly short lengths of stay.

The contrast between per diem payment for SNFs and per episode payments for HHAs translate to contrasting incentives for the intensity of care. Similar to the incentive that hospitals face under the IPPS, HHAs face an incentive to shorten length of stay in PAC and to accept patients with longer length of stay in the hospital. Admitting a patient earlier to these types of PAC erodes margins downstream, as it is costlier to care for patients who are still in intensive stages of recovery. This creates conflicting incentives for hospitals and HHAs, both of which want to shorten length of stay, resulting in an inherent conflict regarding the optimal timing of transition across settings (David et al., 2013).

For SNFs, on the other hand, the per diem payment system creates no incentive to limit PAC length of stay. Admitting a patient earlier from the hospital may or may not erode SNF margins, as margins depend on the relationship between the per diem rates and the costs of care at different points in the patient's trajectory of recovery. Admitting a sicker patient may simply entail a longer length of stay in PAC. The inherent incentive to keep patients in SNFs longer may be balanced in part by the requirement to document the patient's ongoing need for skilled care or the need to have beds available for new patients, potentially to satisfy demand by hospitals as the ongoing source of referrals. In addition, Medicare requires a large co-payment by the patient after the 20th day of SNF care, so patient demand may limit stays past that point. Overall, the contracting problem between hospitals and SNFs in the absence of vertical integration is different from that of HHAs, with less clear incentives with respect to the payment system alone.

Beyond the payment considerations, hospitals and PAC providers have historically had little incentive to coordinate services across settings. Indeed, in some cases, there have been financial incentives for uncoordinated care, such as the case of hospital readmissions, where 
hospitals got reimbursed for a second stay and PAC providers could have multiple episodes of care.

\subsection{Vertical integration}

Vertical integration of hospitals and PAC providers joins the utility function across the two providers:

$$
\max _{I_{h}+I_{P A C}} U_{I_{h}+I_{P A C}}\left[\pi_{I_{h}}+I_{P A C}\left(I_{I_{h}}+I_{P A C}\right), Q_{I_{h}+I_{P A C}}\left(I_{I_{h}+I_{P A C}}\right)\right]
$$

Joint ownership gives financial incentives for hospitals to consider payment and outcomes across the two settings and to allocate general tasks (i.e., tasks that can be performed in either care setting, such as recovery tasks) to their own PAC in a manner that is more efficient (conditional on payment structure), maximizing Medicare payments and patient outcomes. Given that this setting is subject to imperfect and asymmetric information, this task allocation is likely to be more efficient under vertical integration than under market transactions.

In the case of vertical integration between hospitals and HHAs, where both the hospital and the HHA are subject to a predetermined episodic payment, little room is left for manipulating Medicare payments. Gains from efficiency must rely on reducing and reallocating inputs, i.e. reducing joint costs. Vertical integration is still likely to produce efficiencies, but not necessarily efficiencies that result in higher Medicare payment to the combined organization, given the payment structure.

In the case of vertical integration between hospitals and SNFs, however, any shifting of tasks from the hospital to the SNF creates additional payments in addition to any input-related efficiency gains. Even in the absence of input-related efficiency gains, hospitals that vertically integrate with SNFs may benefit from extending SNF length of stay, as they not only gain payment but can also reduce the risk of readmission. A vertically integrated pair can more efficiently balance the costs and benefits of early discharge from the hospital, extended length of stay in the SNF, and reduced readmissions. In addition, in the absence of vertical integration, SNFs may be reluctant to extend length of stay not only due to patient pressure for discharge before high copayments begin but also because of the need to free up beds for discharges. Under vertical integration, the need for beds is subject to more coordination between the SNF and a single hospital, reducing the need for slack. Less pressure to minimize PAC length of stay may enable a stronger focus on using vertical integration to improve patient outcomes that are strategically important to the hospital, such as readmissions.

In this setting with imperfect and asymmetric information, it is also possible that the underlying changes to agency inherent in vertical integration may reduce incentives for quality. As a separate entity, a PAC provider is incentivized through profit motives to provide a level of quality sufficient to attract discharges from hospitals and to minimize the cost of doing so. As part of a vertically integrated organization, PAC managers may face different 
incentives, depending on the structure of payment imposed by the hospital. Incentives for quality and efficiency may be attenuated if the structure of payment is not well aligned with the hospital's goals. However, the hospital's incentive to reduce readmissions - and therefore the incorporation of these incentives into the internal payment structure - is likely to be strong enough to counter reduced agency induced by the change in organizational structure.

Increased market power under vertical integration reinforces the incentive for hospitals to consider payment and outcomes across the two settings and to allocate general tasks to their own PAC in a manner that is more efficient, maximizing Medicare payments and patient outcomes. In this setting, vertical integration can enhance market power by ensuring access to PAC beds for the hospital and ensuring a source of referrals for the PAC. Thus, each faces less competition from other providers who may offer patients or PAC beds that would be more aligned with non-integrated, setting-specific incentives.

\subsection{Informal integration}

Under informal integration, hospitals and PAC providers conduct a significant number of transactions with each other, sometimes but not always reinforced by agreements or contracts to coordinate care, but remain legally separate entities with separate financial structures. Thus, the utility functions of hospitals and PAC providers remain separate. However, interdependence of the two entities (for patient referrals and PAC beds) implies that utility of one provider is taken into account in the utility function of the other:

$$
\begin{gathered}
\max _{I_{h}} U_{h}\left[\pi_{h}\left(I_{h}\right), Q_{h}\left(I_{h}\right), U_{P A C}\left(I_{h}\right)\right] \\
\max _{I_{P A C}} U_{P A C}\left[\pi_{P A C}\left(I_{P A C}\right), Q_{P A C}\left(I_{P A C}\right), U_{h}\left(I_{P A C}\right)\right]
\end{gathered}
$$

Consideration of the other party's utility should lead to more coordination. However, the change in incentives (relative to no integration) faced by hospitals and PAC providers under informal vertical integration are inherently weaker than under formal vertical integration where the utility function is joint. Under informal integration, there is no joint ownership of assets or property rights and no strict hierarchy across organizations in managerial decisions. In addition, increases (or decreases) in revenues are not shared across organizations. Thus, on the margin, more inputs may be invested to increase the profits or quality motives of the other party in conjunction with the effort to improve coordination, but as the benefit from doing so is indirect, incentives for change are small.

In combination, this framework leads to several testable hypotheses, which we address empirically in the next section. 
Hypothesis 1-Vertical integration between hospitals and PAC providers leads to increased Medicare payments for hospital-SNF pairs but not for hospital-HHA pairs.

Hypothesis 2-Vertical integration between hospitals and PAC providers leads to reduced hospital readmissions.

Hypothesis 2A-This effect is likely to be larger for hospital-SNF pairs than for hospitalHHA pairs.

Hypothesis 3-Effects of informal integration will be smaller than of formal vertical integration.

\subsection{Heterogeneity}

Theoretically, the effects of integration may also vary by important hospital and patient characteristics. The effects of integration may be stronger in for-profit than not-for-profit hospitals, as not-for-profit hospitals are more likely to include non-financial considerations in their objective function which may dampen their reaction to financial incentives. We also expect the effects of integration to be strongest for diagnosis groups with the highest payments and where the payments are most variable, as those groups are where efficiency gains are likely to be largest, compared to diagnosis groups with the lowest payments and low variation in payments.

\subsection{Endogeneity}

Estimating the causal relationship between integration and patient outcomes is complicated by at least two potential sources of endogeneity. First, a provider's decision to integrate may related to unobservable attributes of providers that influence both the decision to integrate and patient outcomes. For example, well-managed or more efficient PAC providers may be more attractive integration partners for hospitals, and these same attributes lead to better patient outcomes. Second, patient selection of integrated providers may be non-random and at least partially driven by patient characteristics that are unobservable to the researcher. For example, patients with more complex needs (which are only partly measurable) may search out integrated academic medical centers. Both types of endogeneity need to be addressed empirically in order to obtain unbiased effects of vertical integration on patient outcomes.

\section{Data, sample, and empirical strategy}

\subsection{Data}

Our study relies on patient hospital discharge-level Medicare claims data to observe all Medicare-reimbursed hospitalizations and post-acute care use in the U.S. between 2005 and 2013. We supplement this discharge-level data with Medicare provider-level data for hospitals, SNFs, and HHAs, which are used to measure vertical integration between hospitals and PAC providers. These data sources are described more fully below.

Our primary data source is hospital discharge-level Medicare claims, including the $100 \%$ MedPAR file (including claims for all acute inpatient hospitalizations and SNF stays) and 
home health claims for all patients discharged from an acute care hospital during our study period. The Medicare claims data are supplemented with the Medicare Beneficiary Summary File, which contains information on beneficiary enrollment in Medicare Part A and Part B, demographics including date of birth, sex, and race, and date of death. Medicare claims are used to measure patient outcomes of interest as well as patient discharge-level covariates. In addition, claims are used to develop a measure of informal integration between hospitals and post-acute care providers (described in detail below).

These patient discharge-level data are supplemented with provider-level data from two annual files. These are the Provider of Service (POS) File, containing information on ownership status (e.g. whether a hospital and PAC provider share ownership); and Medicare Cost Reports, containing the linkages between the specific hospitals and other providers that share ownership.

\subsection{Study sample}

We start by including all U.S. acute care hospitals and all Medicare-certified PAC providers. This includes 4011 hospitals, 16,251 SNFs, and 12,720 HHAs. We pair each hospital in a Hospital Referral Region (HRR) with each SNF and HHA in that HRR. We consider these the potential hospital-PAC relationships that exist within health care markets. This results in 146,387 hospital-SNF pairs and 104,253 hospital-HHA pairs. For each PAC type, we restrict our sample of hospital-PAC pairs to those HRRs that have at least one vertically integrated hospital-PAC pair and one informally integrated hospital-PAC pair for each PAC type. ${ }^{1}$ This results in a provider-level sample that includes 109,023 hospital-SNF pairs and 74,597 hospital-HHA pairs. The sample includes $78 \%$ of HRRs in the SNF sample and $85 \%$ in the HHA sample.

We then define our patient sample within the sample of hospital-PAC pairs. The patient discharge-level sample includes all Medicare fee-for-service beneficiaries discharged from an acute care hospital to PAC. We define discharge to PAC as those hospital discharges having a PAC claim within 3 days of hospital discharge. From this sample we exclude all beneficiaries enrolled in Medicare Advantage (MA), as these beneficiaries have incomplete data in the Medicare claims. We exclude beneficiaries if they are enrolled in MA in the one year prior to hospital discharge (to completely measure patient comorbidities) or the 60 days after hospital discharge (to completely measure patient outcomes). We also exclude beneficiaries who are not eligible for Medicare's hospital readmission measure, which is one of our main outcomes. This excludes beneficiaries discharged from the hospital against medical advice, admitted to a PPS-exempt cancer hospital, or with hospital admissions for cancer treatment, primary psychiatric disease, rehabilitation care, or fitting of prostheses and adjustment devices. Finally, because the complete cohort of patients using PAC is very heterogeneous in their primary reasons for hospitalization, we exclude hospitalizations that were not for one of the 25 most common hospital diagnosis related groups (DRGs) for each

\footnotetext{
${ }^{1}$ This restriction of having at least one integrated pair for each PAC type is necessary to calculate our instrumental variable, which is based on differential distance between integrated and non-integrated hospitals within HRR (see below for details). We further restrict to HRRs that have both a vertically integrated and an informally integrated PAC pair for each PAC type to keep our sample consistent between analyses of vertical integration and informal integration.
} 
PAC type. This creates a more homogenous and consistent study population. Approximately $50 \%$ of PAC users have a hospitalization for one of the 25 most common DRGs. Our final sample includes 2,651,748 beneficiaries discharged from hospital to SNF and 1,318,577 discharged to HHA.

\subsection{Integration}

We measure integration between hospitals and PAC providers in two ways. First, we measure formal vertical integration as ownership of a SNF and HHA by an acute care hospital. Vertical integration is measured as a binary variable in each year for every hospitalPAC pair in an HHR. Second, we measure informal integration based on patient flows and the concentration of relationships between hospital and PAC providers. Using Medicare claims data, for each hospital in each HRR we measure the hospital's concentration of discharges to PAC providers in its market using the Herfindahl-Hirschman Index (HHI). To do so, for each PAC type we calculate the share of a hospital's patients going to each PAC provider of that type in its HHR in each year of the study period and then take the sum of squares of those shares, excluding hospitals with fewer than five discharges to a PAC setting. We then identify hospitals with an HHI in the highest quartile (compared to other PACs of the same type across the entire study period). We then pair those concentrated hospitals with the PAC provider to which the hospital sends the plurality of its patients, ${ }^{2}$ resulting in a binary variable indicating whether a hospital-PAC pair is informally integrated, measured annually. The measure is intended to reflect that the hospital and PAC provider have strong ties and interdependence, which may be accompanied by efforts to coordinate care or smooth transitions. ${ }^{3}$ We create this measure of informal integration separately for each of the two PAC types. We check the sensitivity of this measure to small changes in patient flows in two ways. First, we check the length of time that informally integrated hospital-PAC pairs are identified as informally integrated and find that hospital-SNF pairs are identified as such on average for 5.5 of the 9 years of the study and informally integrated hospital-HHA pairs are identified as such for 6.2 years on average. Second, we confirm that the most of these pairs stay informally integrated for the majority of the informal integration spell (rather than moving in and out of being informally integrated) and find that when hospitalSNF pairs are informally integrated, they are such continuously $85 \%$ of the time; and hospital-HHA pairs are $84 \%$ of the time.

These two measures of integration are summarized in Figs. 1 and 2, showing trends in integration between hospital-PAC pairs over our study period. Rates of both formal and informal integration declined over the study period for SNFs and HHAs. ${ }^{4}$ Table 1 shows the overlap between these two measures of integration. The majority of integrated provider pairs are either vertically integrated or informally integrated, but not both. For example, for hospital-SNF pairs, $1.0 \%$ of pairs are vertically integrated and $1.2 \%$ are informally

\footnotetext{
2 If the two most commonly used PAC providers have shares that are within 5 percentage points of each other, we consider it a tie and match the hospitals with two PAC providers.

${ }^{3}$ Alternative definitions of informal integration based on the importance of the hospital-PAC relationship from the perspective of the PAC provider yielded very similar results.

${ }^{4}$ Over the study period, $3.0 \%$ of SNFs changed vertical integration status and $10.6 \%$ changed informal integration status. For HHAs, $6.0 \%$ and $10.8 \%$ changed integration, respectively.
} 
integrated. Only $0.3 \%$ of all hospital-SNF pairs are both vertically and informally integrated while $0.8 \%$ are only vertically integrated and $0.9 \%$ are only informally integrated.

\subsection{Outcome variables}

We measure the impact of integration on three patient discharge-level outcomes. First, we examine Medicare payments over an episode of care encompassing both acute and postacute care, defining the episode as 60 days from the date of admission to the hospital. ${ }^{5} \mathrm{We}$ include three measures of Medicare payments: 1) the sum of Medicare payments for the index hospitalization and for the first PAC stay during the 60-day time period; 2) Medicare payments for the index hospitalization; and 3) Medicare payments for the first PAC stay.

Second, we examine length of stay. Like Medicare payments, we measure length of stay in three ways: for the entire 60-day acute/post-acute care episode and also separately for acute care and for post-acute care. For both payments and length of stay we account for censoring by death in all regressions by including two covariates: a dummy variable indicating whether the patient died in the first 60-days and the number of days censored due to death.

Our third outcome is readmission to the hospital within 30 days of hospital discharge. We follow Medicare's definition of hospital readmission from the Hospital Readmission Reduction Program, which includes readmissions to any acute care hospital within 30 days of discharge from an index hospitalization where the readmission was not planned. ${ }^{6}$ We use a combined endpoint of readmission or death within 30 days of hospital discharge to account for censoring by death.

Patient outcomes in our study cohort are summarized in Table 2.

\subsection{Other variables}

In addition to the instrumental variables approach (described below) used to account for observed and unobserved differences in patient case-mix across hospitals and PAC providers, in all regressions we account for residual heterogeneity by including measures of age, sex, race, 31 indicators for comorbidities, ${ }^{7}$ and indicators for each of the 25 DRGs included in the study sample. ${ }^{8}$ These covariates are summarized in Table 3.

\subsection{Empirical strategy}

We estimate empirically the effect of changes in the integration status of hospital and PAC providers on Medicare costs and patient outcomes. We do so using a provider fixed-effects

\footnotetext{
${ }^{5}$ We define this outcome over 60 days because $90 \%$ of hospital-PAC episodes in our data are less than 60 days and thus are encompassed within the 60-day episode.

${ }^{6}$ Planned readmissions include those for bone marrow or solid organ transplant, maintenance chemotherapy, rehabilitation, or a potentially planned procedure not performed to treat an acute condition or a complication of previous care.

${ }^{7}$ Comorbidities are defined based on CMS Hospital Readmission Reduction Program specifications for risk adjustment of readmission and include: severe infection; other infectious disease pneumonias; metastatic cancer/acute leukemia; severe cancer; other cancers; diabetes mellitus; protein-calorie malnutrition; end-stage liver disease; severe hematological disorders; alcohol abuse; psychiatric comorbidity; hemiplegia, paraplegia, paralysis, functional disability, seizure disorders, CHF, coronary atherosclerosis or angina/CVD; specified arrhythmias; COPD; fibrosis of lung or other chronic lung disorders; dialysis; decubitus ulcer or chronic skin ulcer; septicemia/shock; disorders of fluid, electrolyte, acid-base; iron deficiency or other unspecified anemias; cardiorespiratory failure or shock; acute renal failure; pancreatic disease; rheumatoid arthritis; respiratory dependence/tracheostomy; transplant; coagulation defects/hematologic disorders; hip fracture/dislocation.

${ }^{8}$ See Appendix Table 1 in Supplementary material for the list of the top 25 DRGs in each PAC setting.
} 
model, leveraging longitudinal data from 2005 to 2013. In these data we observe annual integration status for every hospital-PAC pair in our sample over time, identifying the effect of integration by changes in integration status within hospital-PAC pair. Our basic model specification is:

$$
Y_{i j t}=\beta_{1} \text { Integration }_{j t}+X_{i t}+\mu_{j}+\varphi_{t}+\varepsilon_{i j t}
$$

where $i$ indexes the hospital discharge, $j$ indexes the hospital-PAC pair, and $t$ indexes year. The dependent variable, $Y_{\mathrm{ijt}}$, is one of the patient discharge-level variables defined above (Medicare costs, length of stay, or readmission/death). The independent variable of interest is Integration $n_{\mathrm{jt}}$, which is a dummy variable measuring integration either formally (vertical integration) or informally (based on patient flows) at the hospital-PAC pair level in each year. We also include patient discharge-level covariates, hospital-PAC pair fixed effects, and year fixed effects. We estimate this equation separately for formal and informal integration and for SNFs and HHAs.

As discussed in the conceptual model section, estimating the effect of integration on patient outcomes may be biased from at least two potential sources of endogeneity. First, a provider's choice to integrate may be endogenous to patient outcomes. We rely on providerpair fixed effects to address this potential source of endogeneity. By including provider-pair fixed effects we difference out any time-invariant environmental factors at a hospital-PAC pair that affect integration decisions and patient outcomes, estimating within-provider effects of changing integration status on patient outcomes.

Second, patient selection of integrated hospitals may be nonrandom and at least partially based on patient characteristics that are unobservable to the researcher. To account for this potential endogeneity, we estimate an instrumental variables model. We use differential distance as our instrument, defining differential distance as the difference in miles between the distance from the centroid of a patient's home ZIP code to the nearest integrated hospital and the nearest non-integrated hospital in miles, measured separately for vertical integration and informal integration, and for each PAC type. This differential distance encompasses both positive and negative values, depending on whether the beneficiary's home is closer to an integrated or a non-integrated hospital. A summary of the differential distance to hospitals integrated with SNFs and HHAs in our sample is displayed in Table 4. In all cases, the mean differential distance is positive, indicating the higher prevalence of non-integrated hospitals.

Differential distance is a valid instrument if it is strongly correlated with a patient's choice of an integrated hospital but not $\varepsilon$, the error term in Eq. (6). To test the strength of the correlation we use the standard test of Staiger and Stock (1997), examining whether the $F$ statistics exceed the threshold of 10 (or $t$-statistics exceeding 3.16 for a single instrument). The first-stage estimates in Table 5 show that the correlations far exceed this threshold, with $F$-statistics of 126.6 and 215.8 for the instrument of differential distance to a vertically integrated SNFs and HHAs respectively and 428.9 and 508.1 for differential distance to an informally integrated SNFs and HHAs. 
While we cannot test the second assumption of our instrument having no correlation with the error term directly, we can examine whether our instrument is correlated with observed patient characteristics. To do so, we divide differential distances at the median and examine patient characteristics for those beneficiaries with a differential distance above and below the median (or near versus far from an integrated hospital). These results are displayed in Appendix Table 2 in Supplementary material, showing that in general patient discharge-level characteristics are very similar across these two groups, suggesting that the samples are balanced. One exception to this balance is the differential distance to hospitals that are vertically integrated and informally integrated with SNFs by race, where white beneficiaries tend to have a longer differential distance while blacks and other beneficiaries who are nonwhite have a shorter differential distance. We include race as well as these other observable characteristics as controls in our models.

We examine Eq. (6) using a linear probability model. All standard errors are adjusted for heteroscedasticity using the Huber-White estimator of variance clustered at the provider-pair level.

\section{Results}

\subsection{Primary findings}

Estimates of the effect of integration on Medicare payments are presented in Table 6. Hospital-SNF pairs that are vertically integrated receive higher total Medicare payments for the first 60 days of care than hospital-SNF pairs that are not vertically integrated by $\$ 2424$ on a base of $\$ 14,291$ (or a relative increase of $17.0 \%$ ). This is driven by higher payments to PACs, with vertically integrated SNFs receiving \$2274 more than non-integrated SNFs, whereas payments to hospitals are similar by integration status. On the other hand, for vertically integrated HHAs, vertical integration is associated with lower total Medicare payments over the first 60 days, though the magnitude of the effect is smaller. Vertically integrated hospital-HHA pairs experience a reduction of \$303 in total Medicare payments on a base of $\$ 10,352$ (for a relative reduction of $2.9 \%$ ).

The effects of informal integration on total Medicare payments are generally small and not statistically significant. We do observe a statistically significant reduction in hospital payments for hospitals that are informally integrated with a SNF, but of only $\$ 326$ (or a relative reduction of $2.2 \%$ for the average hospital) and with no change in total Medicare payment.

We next examine how length of stay changes in integration to help provide some context for payment changes, presented in Table 7. The total length of stay for the initial hospital and PAC stay increases for hospital-SNF pairs that are vertically integrated by 3.9 days on a base of 30.5 days (or by $12.8 \%$ ). This change is largely driven by increasing length of the SNF stay with a decline in hospital length of stay. Hospitals that are vertically integrated with HHAs experience a decline in length of stay, which is driven by a decline in the length of HHA episodes. 
Among informally integrated pairs the effects of integration on length of stay are generally small and not statistically significant. The one exception to this is for hospital-SNF pairs that are informally integrated where we observe a decline in length of stay that is driven by a shorter SNF stay.

Finally, we test whether integration affects patient outcomes (see Table 8). We estimate that hospitals that are vertically integrated with SNFs have a decline in their rate of 30-day readmission or death by just over 5 percentage points on a base rate of $31.3 \%$ (for a relative decline of $17 \%$ ). For vertically integrated HHAs the effect of integration on readmission or death is close to zero. Estimates of the effect of informal integration between hospitals and PAC providers on readmission or death are small and not statistically significant. ${ }^{9}$

\subsection{Heterogeneous effects}

We test whether the effects of integration vary by important hospital and patient characteristics. First, we stratify our estimation by hospital profit status, hypothesizing that the effects of integration will be stronger in for-profit than not-for-profit hospitals. We find this to be the case in hospitals that are vertically integrated with SNFs across all of our outcomes (see Table 9). The effects are also somewhat stronger among for-profit hospitals that are vertically integrated with HHAs (see Table 10), as compared to vertically interated non-profit hospitals. As in our main specifications we do not see a strong effect among hospitals that are informally integrated with SNFs or HHAs.

We also stratify by patient diagnoses, focusing on diagnosis groups with the highest payments and where the payments are most variable, compared to diagnosis groups with the lowest payments and low variation in payments. These are also detailed Tables 9 and 10. Among hospitals that are vertically integrated with SNFs, we generally find that Medicare SNF payment and length of stay for SNF increase more significantly for high-cost/highvariance conditions compared to low-cost/low-variance conditions, with some mixed results. For vertically integrated HHAs, the effect of vertical integration on HHA payment and LOS are larger in high-cost/high-variance conditions.

\subsection{Robustness checks}

In order to test the robustness of our results to primary model specification, variable definition, cohort definition, we run a series of robustness checks. These are presented in Appendix Tables 3-5 in Supplementary material. First, because some hospital-PAC pairs are both vertically integrated and informally integrated, we limit our analyses to those hospitalPAC pairs that are either vertically integrated or informally integrated. When we rerun the model after excluding pairs that are both vertically and informally integrated, we find stronger effects of vertical integration on almost all outcome variables. The effect of informal integration on these outcomes remains small and statistically insignificant in most cases.

\footnotetext{
${ }^{9}$ These primary findings were substantially different when we did not include provider-pair fixed effects in the estimation, suggesting that provider-level endogeneity is important to account for. These results are available from the authors upon request.
} 
A second potential concern is that the effects of vertical integration may be concentrated in the most competitive markets. Thus, we restrict our sample to the most competitive markets. Using the Hospital Referral Region to approximate market, we calculate market concentration as the HHI based on each hospital's share of beds in the market. We then estimate the effects of integration on the sample with an HHI of less than 2500. This restriction does not qualitatively change the results.

Third, entry or exit of providers over time may affect the estimation. We re-estimate the model on the balanced subsample of hospital-PAC pairs so that the same cohort of hospitalPAC pairs is observed in each of the nine years of the study time frame. While this excludes a large number of hospital-PAC pairs, the total number of hospital discharges captured in this smaller sample is $83 \%$ of the original sample. This suggests that this restriction primarily excludes hospital-PAC pairs that share relatively few patients and thus are likely to drop out of the sample in periods in which they share no patients. Our results are robust to this sample exclusion, indicating that the results are not driven by a changing sample of providers over the study period.

We also recalculate total Medicare payments to include all institutional Medicare payments in the first 60 days after hospital admission. Unlike the dependent variable in the primary specification, this variable includes Medicare payments for rehospitalizations as well as any additional payments for PAC after the index PAC stay. Using this variable as the dependent variable produces similar estimates of the effects of integration.

We include a specification check on functional form in the length of stay analyses. Because this is a count variable, we re-estimate the main regression using a negative binomial model. While we do find some minor changes in statistical significance with our specification checks, in all cases the results remain qualitatively similar.

For the analyses of informal integration, we perform two additional robustness tests. We restrict our measure of informal integration to only those hospitals with at least 25 discharges to a PAC setting (rather than limiting it to those hospitals with at least 5 discharges as we did in our primary specification). In our primary measure of informal integration, a high proportion of small hospitals were identified as being highly concentrated in their PAC discharges and thus, in informally integrated relationships with PAC providers. By limiting the sample to larger hospitals, we aim to ensure that our results are not driven by small hospitals. This exclusion does not affect our findings.

We also consider a less strict definition of informal integration. As in our primary definition of informal integration, we measure the concentration of hospital discharges to SNF but focus on hospitals with a lower discharge centration, using an absolute threshold of greater than or equal to $3800 .{ }^{10}$ We then pair those concentrated hospitals with the PAC provider to which the hospital sends the plurality of its patients. This change in the definition of informal integration results in slightly larger estimates of the effect of informal integration

$10_{3800}$ was chosen as it is the median HHI across SNFs and HHAs combined. This lowers the hospital HHI from the 75 th percentile in our original specification to the 60th percentile in SNFs and the 40th percentile in HHAs. 
on SNF payment, HHA payment, and HHA length of stay. However, the effect of informal integration remains substantially smaller than the effect of vertical integration.

\section{Conclusion}

In this study, we present empirical estimates of the effects of hospital-PAC integration on Medicare spending and patient outcomes. These estimates leverage longitudinal data, better measurement than in prior studies, and an instrumental variables approach to provide a more complete picture of the effects of integration in PAC across the full array of PAC settings, and a range of outcomes and patient samples that are relevant to current policy debates.

Our findings are consistent with the hypothesis that the effect of vertical integration depends on the payment mechanism for PAC - whether it is per-diem or episode-based paymentand that informal integration will have a smaller impact on health care delivery and patient outcomes. We find that in vertically integrated SNFs, where the hospital-SNF pairs have incentive to lengthen SNF stays to maximize Medicare payment, SNF length of stay and Medicare payments to SNF increase. This is accompanied by a reduction in rehospitalization rates. In contrast, in vertically integrated HHAs, where HHAs are paid by episode, vertical integration is associated with at most a small reduction in Medicare payment and no change in rehospitalization rates. Under informal integration, where hospital-PAC pairs face weaker incentives for coordination than vertical integration, we find little effect on either Medicare payment or patient outcomes in most specifications.

The overall increases in SNF length of stay that we observe among vertically integrated hospital-SNF pairs is the combined effect of a small decrease in hospital length of stay and a larger increase in SNF length of stay. The decrease in hospital length of stay provides some evidence that vertical integration has induced more efficient task allocation between hospitals and SNFs (David et al., 2013). That is, hospitals shift the management of patients from hospitals to SNFs sooner (where the production of care is less expensive), and SNFs have incentive to accept patients quicker and sicker because they can share in the joint revenue from the hospital stay. The increase in SNF length of stay is in part due to accepting patients quicker and sicker, and we do not expect a one-to-one tradeoff between a hospital day and a SNF day since SNFs provide lower intensity of services. Whether the increased SNF length of stay is due only to accepting patients quicker and sicker is not known from our data as they do not contain provider costs.

From a hospital's perspective, vertical integration should increase profits to hospitals through lower costs from decreased length of stay, though we do not have measures of hospital costs or profits in our data. SNFs will also generate more revenue under vertical integration from longer lengths of stay. However, the costs of taking care of sicker patients will be higher, and the SNF stay may need to be longer to recover those costs. While we cannot directly assess whether this increase in revenues covers the costs of caring for sicker patients, the observation that SNFs increase lengths of stay provides evidence that there are financial benefits to the hospital-SNF pair from doing so. 
From Medicare's perspective, costs increase under vertical integration, but there is some value from this in reduced readmission rates. The magnitudes of the effects we estimate for changes in SNF payments are meaningful. With vertically integrated hospital-SNF pairs receiving over $\$ 2400$ more per beneficiary from Medicare than non-integrated pairs, this $17 \%$ relative increase in payment is large but not out of proportion to the estimated increase in SNF length of stay. This increase in spending is accompanied by a significant reduction in rehospitalization rates of over 5 percentage points. Despite this reduction in rehospitalization rates, total spending by Medicare remains higher among beneficiaries receiving care in vertically integrated hospital-SNF pairs. Summing the mean per-beneficiary Medicare payment for index hospitalizations, readmissions, and SNF stays, Medicare appears to be spending $11 \%$ more on vertically integrated care compared to non-vertically integrated care. 11 The total cost to Medicare of vertical integration depends on how widespread vertical integration between hospitals and SNF is, but based on the over 1.1 million beneficiaries we observe using SNF annually in our data, the Medicare cost could range from \$209 million annually if $10 \%$ of hospital-SNF pairs were vertically integrated to over $\$ 2$ billion annually of all SNFs were vertically integrated with hospitals.

While the reduction in rehospitalizations is an important outcome, it comes at a high price under vertical integration. This raises the question of whether there are alternative ways to improve coordination between hospitals and PAC to improve patient outcomes. We find that informal integration has little effect on Medicare payment or on patient outcomes. While this may not be surprising given the weak financial incentives that informal integration provides to coordinate care, informal integration is frequently cited in the trade literature as a way to improve care coordination, decrease spending, and prepare for payment reforms that hold providers accountable for the costs and quality of care. Numerous case studies describe the formation of networks and other forms of informal integration between hospitals and postacute care providers with the goal of improving patient transitions of care and positioning providers to be competitive under payment reform. As one trade publication advises: "Feefor-value reimbursement, like bundled payments, and payment penalties relating to hospital readmissions, will require hospitals and post-acute care ('PAC') providers to be better integrated in order to control costs and maintain positive margins"(Baylor Quality Alliance, 2012).

Despite the positive view of integration described in the trade literature, we do not see evidence of an increased trend to informal integration in our data, and we estimate no significant benefit when it does exist. While our data goes through 2013, it is possible that this trend toward integration is more recent and we therefore miss it in our data.

Finally, it is possible that payment reform will successfully alter the incentives in the production of PAC to improve patient outcomes at a lower cost than vertical integration. Early data on the effects of ACOs suggest that Medicare ACOs result in reduced Medicare spending on SNFs (McWilliams et al., 2017). However, these ACOs have not shown an

\footnotetext{
${ }^{11}$ For a vertically integrated episode of care, Medicare spends approximately $\$ 8042$ for a hospitalization, $\$ 1834$ for a readmission (or the cost of a hospitalization (\$8042) times the probability of readmission (22.8\% excluding deaths)), and \$6249 for a SNF stay. For non-vertically integrated episodes, the Medicare payment for a hospitalization remains the same at $\$ 8042$, but payments for rehospitalizations go down to $\$ 1399$ ( $\$ 8042 * 17.4 \%$ ), and SNF payments go up to $\$ 8522$.
} 
appreciable effect on readmission rates (McWilliams et al., 2016). This research has not assessed the effects of ACOs on the level of integration between hospitals and PAC providers. Under payment reforms that increasingly hold providers accountable for the costs of care, it is possible that pressure to more effectively integrate using informal mechanisms will increase and will provide stronger incentives to meaningfully coordinate care. Future research might better tease out the interaction between payment reform and different types of integration.

While our results add to the existing literature on integration by providing robust estimates of both vertical and informal integration that account for patient and provider selection, we must be care ful in interpreting these results. To account for patient selection, we use an instrumental variable approach. This provides the local average treatment effect of integration, where the marginal patients are those who went to an integrated hospital simply because they lived closer to an integrated hospital than a non-integrated hospital. These patients may not be representative of patients who choose to go to an integrated hospital for other reasons. To account for provider selection, we use a longitudinal fixed-effect model, identifying the effect of integration from hospital-PAC pairs that had a change in integration over the study period. These hospital-PAC pairs may not be representative of those integrated pairs that did not change their integration status. Nonetheless, among those hospital-PAC pairs that were integrated at the start of the study period, a large portion changed their integration status over the course of the subsequent nine years, and we provide robust estimates of the effect of those changes.

This study provides new estimates of the effects of vertical integration between hospitals and PAC. From a more fundamental perspective our study emphasizes that providers respond to the financial incentives they face and, thus, the details of payment mechanisms have important impacts on the organization of health care delivery and on provider behavior. In promoting policies to increase coordination of care, policymakers should bear in mind that integration may be inherently anti-competitive and that coordination may be accompanied by higher spending. Thus, designing financial incentives to increase coordination of care and simultaneously control costs are challenging. As we move forward with payment reform aimed at constraining costs and improving quality, designing reforms that anticipate provider responses will be key to their success.

\section{Supplementary Material}

Refer to Web version on PubMed Central for supplementary material.

\section{Acknowledgments}

This work was supported by a Health Care Financing and Organization grant from the Robert Wood Johnson Foundation. Rachel Werner was supported in part by grant K24-AG047908 from the NIA.

\section{Appendix A. Supplementary data}

Supplementary data associated with this article can be found, in the online version, at https:// doi.org/10.1016/j.jhealeco.2018.01.005. 


\section{References}

Baicker K, Levy H. Coordination versus competition in health care reform. N Engl J Med. 2013; 369(9):789-791. [PubMed: 23944255]

Baker LC, Bundorf MK, et al. Vertical integration: hospital ownership of physician practices is associated with higher prices and spending. Health Aff (Millwood). 2014; 33(5):756-763. [PubMed: 24799571]

Baylor Quality Alliance. Integrating Acute and Post-acute Care: The Emerging Merging of The Sectors. Strategies for Health Care Leaders. 2012; 71

Beers M, Sliwkowski J, et al. Compliance with medication orders among the elderly after hospital discharge. Hosp Formul. 1992; 27(7):720-724. [PubMed: 10119187]

Burns LR, Muller RW. Hospital-physician collaboration: landscape of economic integration and impact on clinical integration. Milbank Q. 2008; 86(3):375-434. [PubMed: 18798884]

Centers for Medicare and Medicaid Services. Medicare \& Medicaid Statistical Supplement. 2007

Centers for Medicare and Medicaid Services. Shared Savings Program. 2013. From http:// www.cms.gov/Medicare/Medicare-Fee-for-Service-Payment/sharedsavingsprogram/index.html? redirect=/sharedsavingsprogram/

Centers for Medicare and Medicaid Services. Readmission Reduction Program. 2013. Retrieved 9/27/2013, from http://www.cms.gov/Medicare/Medicare-Fee-for-Service-Payment/ AcuteInpatientPPS/Readmissions-Reduction-Program.html

Chandra A, Dalton MA, et al. Large increases in spending on postacute care in Medicare point to the potential for cost savings in these settings. Health Aff (Millwood). 2013; 32(5):864-872. [PubMed: 23650319]

Ciliberto F, Dranove D. The effect of physician-hospital affiliations on hospital prices in California. J Health Econ. 2006; 25(1):29-38. [PubMed: 16352360]

Coleman EA, Berenson RA. Lost in transition: challenges and opportunities for improving the quality of transitional care. Ann Intern Med. 2004; 141(7):533-536. [PubMed: 15466770]

Coleman EA, Mahoney E, et al. Assessing the quality of preparation for posthospital care from the patient's perspective: the care transitions measure. Med Care. 2005a; 43(3):246-255. [PubMed: 15725981]

Coleman EA, Smith JD, et al. Posthospital medication discrepancies: prevalence and contributing factors. Arch Intern Med. 2005b; 165(16):1842. [PubMed: 16157827]

Congressional Budget Office. Medicare Spending on Post-acute Care: A Preliminary Analysis. Congressional Budget Office; Washington, D.C: 1997.

Cuellar A, Gertler P. Strategic integration of hospitals and physicians. J Health Econ. 2006; 25(1):1. [PubMed: 16309766]

Cutler DM, Scott Morton F. Hospitals, market share, and consolidation. JAMA. 2013; 310(18):19641970. [PubMed: 24219952]

David G, Rawley E, et al. Integration and task allocation: evidence from patient care. J Econ Manage Strategy. 2013; 22(3):617-639.

Feder J, Hadley J, et al. How did Medicare's prospective payment system affect hospitals? N Engl J Med. 1987; 317(14):867. [PubMed: 3306387]

Gaynor M, Town RJ. Provider competition. In: Borras P, McGuire TE, Pauly M, editorsHandbook of Health Economics. Elsevier; Amsterdam: 2012. 499-637.

Guterman S, Dobson A. Impact of the Medicare prospective payment system for hospitals. Health Care Financ Rev. 1985; 7(3):97-114. [PubMed: 10311401]

Harrison A, Verhoef M. Understanding coordination of care from the consumer's perspective in a regional health system. Health Serv Res. 2002; 37(4):1031-1054. [PubMed: 12236382]

Jencks SF, Williams MV, et al. Rehospitalizations among patients in the Medicare fee-for-service program. N Engl J Med. 2009; 360(14):1418-1428. [PubMed: 19339721]

Kondo KK, Damberg CL, et al. Implementation processes and pay for performance in healthcare: a systematic review. J Gen Intern Med. 2016; 31(Suppl. 1):61-69. [PubMed: 26951276] 
Madison K. Hospital-physician affiliations and patient treatments, expenditures, and outcomes. Health Serv Res. 2004; 39(2):257-278. [PubMed: 15032954]

Manton KG, Woodbury MA, et al. Use of Medicare services before and after introduction of the prospective payment system. Health Serv Res. 1993; 28(3):269-292. [PubMed: 8344820]

McWilliams JM, Hatfield LA, et al. Early performance of accountable care organizations in Medicare. N Engl J Med. 2016; 374:2235-2366. [PubMed: 27042964]

McWilliams JM, Gilstrap LG, et al. Changes in postacute care in the Medicare Shared Savings Program. JAMA Intern Med. 2017; 177:518-526. [PubMed: 28192556]

MedPAC. A Data Book: Health Care Spending and the Medicare Program. Medicare Payment Advisory Commission; Washington, DC: 2013a. June 2013

MedPAC. Report to the Congress: Medicare Payment Policy. Medicare Payment Advisory Commission; Washington, DC: 2013b. March 2013

Moore C, Wisnivesky J, et al. Medical errors related to discontinuity of care from an inpatient to an outpatient setting. J Gen Intern Med. 2003; 18(8):646-651. [PubMed: 12911647]

Neprash HT, Chernew ME, et al. Association of financial integration between physicians and hospitals with commercial health care prices. JAMA Intern Med. 2015:1-8.

Rahman M, Norton EC, et al. Do hospital-owned skilled nursing facilities provide better post-acute care quality? J Health Econ. 2016; 50:36-46. [PubMed: 27661738]

Robinson JC. Administered pricing and vertical integration in the hospital industry. J Law Econ. 1996:357-378.

Staiger D, Stock JH. Instrumental variables regression with weak instruments. Econometrica: J Econ Soc. 1997:557-586.

Van Walraven C, Seth R, et al. Effect of discharge summary availability during post-discharge visits on hospital readmission. J Gen Intern Med. 2002; 17(3):186-192. [PubMed: 11929504]

Van Walraven C, Mamdani M, et al. Continuity of care and patient outcomes after hospital discharge. J Gen Intern Med. 2004; 19(6):624-631. [PubMed: 15209600]

vom Eigen KA, Walker JD, et al. Carepartner experiences with hospital care. Med Care. 1999; 37(1): 33-38. [PubMed: 10413390] 
Vertically Integrated Pairs

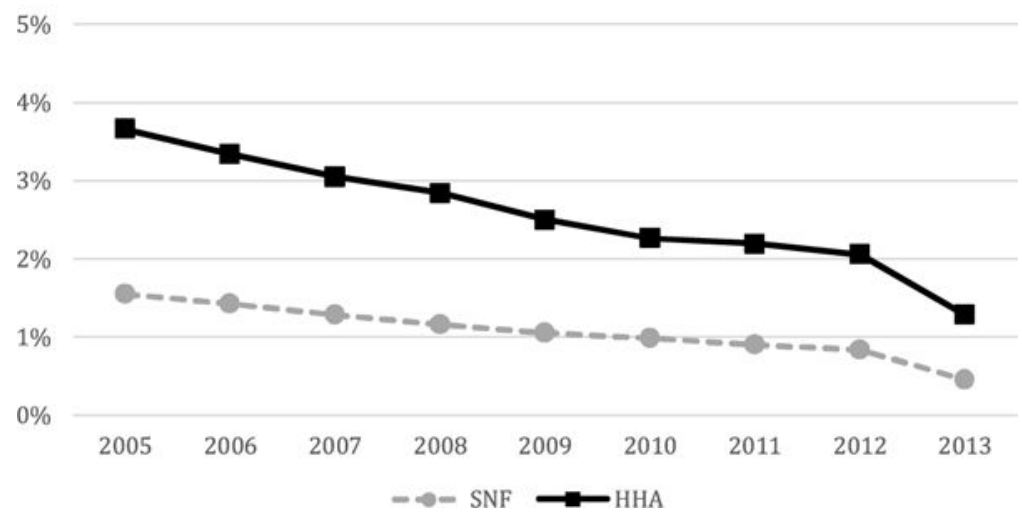

Fig. 1.

Proportion of hospital-PAC pairs that are vertically integrated over the study period. 
Informally Integrated Pairs

$5 \%$

$4 \%$

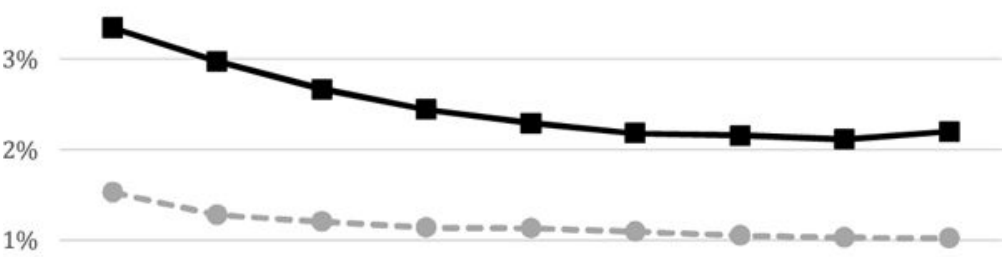

$0 \%$

2005

2006

2007

$2008 \quad 2009$

010

2011

2012

2013

Fig. 2.

Proportion of hospital-PAC pairs that are informally integrated over the study period. 


\section{Table 1}

Percentage of hospital-PAC pairs that are vertically integrated, informally integrated, both, or neither.

\begin{tabular}{lll}
\hline & SNF & HHA \\
\hline All vertically integrated pairs & $1.0 \%$ & $2.5 \%$ \\
All informally integrated pairs & $1.2 \%$ & $2.6 \%$ \\
Both VI and informally integrated & $0.3 \%$ & $0.9 \%$ \\
VI but not informally integrated & $0.8 \%$ & $1.6 \%$ \\
Informally integrated but not VI & $0.9 \%$ & $1.5 \%$ \\
Neither VI nor informally integrated & $98.0 \%$ & $96.0 \%$ \\
\hline
\end{tabular}




\section{Table 2}

Summary of outcome variables.

\begin{tabular}{lll}
\hline & SNF (n= 2,651,748) & HHA (n = 1,318,577) \\
\hline Medicare payment in first 60 days for index hospitalization and PAC stay in \$, mean (SD) & $14,291(8272)$ & $10,352(6508)$ \\
Medicare payment in first 60 days for index hospitalization in \$, mean (SD) & $8042(4908)$ & $7858(6252)$ \\
Medicare payment in first 60 days for index PAC stay in \$, mean (SD) & $6249(6252)$ & $2494(1385)$ \\
Length of stay in first 60 days for index hospitalization and PAC stay in days, mean (SD) & $30.5(17.5)$ & $36.0(17.6)$ \\
Length of stay for index hospitalization in days, mean (SD) & $6.5(4.6)$ & $5.0(3.6)$ \\
Length of stay in first 60 days for index PAC stay in days, mean (SD) & $23.9(17.1)$ & $31.0(17.2)$ \\
30-day rehospitalization or death, n (\%) & $829,506(31.3)$ & $243,688(18.5)$ \\
\hline
\end{tabular}


Table 3

Summary of post-acute care patients.

\begin{tabular}{|c|c|c|}
\hline & SNF $(n=2,651,748)$ & HHA $(n=1,318,577)$ \\
\hline Age, mean (SD) & $82.6(8.2)$ & $79.27(8.2)$ \\
\hline Female, n (\%) & $1,771,929(66.8)$ & $821,266(62.3)$ \\
\hline \multicolumn{3}{|l|}{ Race, $\mathrm{n}(\%)$} \\
\hline -White & $2,298,531(86.7)$ & $1,143,253(86.7)$ \\
\hline -Black & $252,261(9.5)$ & $115,029(8.7)$ \\
\hline -Other & $21,852(0.8)$ & $14,196(1.1)$ \\
\hline -Asian & $32,014(1.2)$ & $15,726(1.2)$ \\
\hline -Hispanic & $34,472(1.3)$ & $22,684(1.7)$ \\
\hline -North American Native & $6478(0.2)$ & $4010(0.3)$ \\
\hline -Unknown & $6140(0.2)$ & $3679(0.3)$ \\
\hline \multicolumn{3}{|l|}{ Comorbidities, $\mathrm{n}(\%)$} \\
\hline -Severe Infection & $35,788(1.3)$ & $12,824(1.0)$ \\
\hline -Other Infectious Disease \& Pneumonias & $824,257(31.1)$ & $268,259(20.3)$ \\
\hline -Metastatic Cancer/Acute Leukemia & $64,705(2.4)$ & $31,676(2.4)$ \\
\hline -Severe Cancer & $107,660(4.1)$ & $56,528(4.3)$ \\
\hline -Other Cancers & $142,306(5.4)$ & $64,325(4.9)$ \\
\hline -Diabetes Mellitus & $823,447(31.1)$ & $398,046(30.2)$ \\
\hline -Protein-calorie Malnutrition & $345,726(13.0)$ & $76,884(5.8)$ \\
\hline -End-stage Liver Disease & $31,035(1.2)$ & $12,268(0.9)$ \\
\hline -Severe Hematological Disorders & $41,528(1.6)$ & $18,770(1.4)$ \\
\hline -Alcohol Abuse & $66,303(2.5)$ & $13,997(1.1)$ \\
\hline -Psychiatric Comorbidity & $667,232(25.2)$ & $236,461(17.9)$ \\
\hline -Hemiplegia, paraplegia, paralysis, disability & $162,315(6.1)$ & $38,703(2.9)$ \\
\hline -Seizure Disorders & $137,133(5.2)$ & $35,251(2.7)$ \\
\hline -Congestive heart failure & $698,022(26.3)$ & $274,544(20.8)$ \\
\hline -Coronary atherosclerosis or angina & $1,375,417(51.9)$ & $624,115(47.3)$ \\
\hline -Specified arrhythmias & $651,168(24.6)$ & $255,648(19.4)$ \\
\hline -Chronic obstructive pulmonary disease & $796,597(30.0)$ & $366,763(27.8)$ \\
\hline -Other chronic lung disorders & $99,699(3.8)$ & $52,112(4.0)$ \\
\hline -Dialysis & $37,859(1.4)$ & $12,002(0.9)$ \\
\hline -Decubitus Ulcer or Chronic Skin Ulcer & $192,132(7.2)$ & $45,613(3.5)$ \\
\hline -Septicemia/Shock & $183,439(6.9)$ & $41,793(3.2)$ \\
\hline -Disorders of fluid, electrolyte, acid-base & 792,087 (29.9) & $257,512(19.5)$ \\
\hline -Iron Deficiency or other anemias & $1,183,565(44.6)$ & $480,113(36.4)$ \\
\hline -Cardiorespiratory Failure or Shock & $282,161(10.6)$ & $99,360(7.5)$ \\
\hline -Acute Renal Failure & $566,091(21.3)$ & $194,542(14.8)$ \\
\hline -Pancreatic Disease & $45,988(1.7)$ & $20,989(1.6)$ \\
\hline -Rheumatoid Arthritis & $112,500(4.2)$ & $56,776(4.3)$ \\
\hline -Respirator Dependence/Tracheostomy & $7989(0.3)$ & $2065(0.2)$ \\
\hline
\end{tabular}

J Health Econ. Author manuscript; available in PMC 2018 September 21. 


\begin{tabular}{lll}
\hline & SNF (n = 2,651,748) & HHA (n = 1,318,577) \\
\hline -Transplants & $5268(0.2)$ & $3747(0.3)$ \\
-Coagulation Defects/Hematologic Disorders & $117,307(4.4)$ & $42,132(3.2)$ \\
-Hip Fracture/Dislocation & $123,631(4.7)$ & $20,189(1.5)$ \\
Six most common reasons for hospitalization, $\mathrm{n}(\%)$ & \\
-Total knee or hip replacement & $352,519(13.3)$ & $288,402(21.9)$ \\
-Sepsis & $213,601(8.1)$ & $59,332(4.5)$ \\
-Congestive heart failure & $259,897(9.8)$ & $177,957(13.5)$ \\
-Urinary tract infection & $211,318(8.0)$ & $61,905(4.7)$ \\
-Hip Fracture & $226,242(8.5)$ & 0 \\
-Pneumonia & $215,725(8.1)$ & $107,369(8.1)$ \\
\hline
\end{tabular}

-Coagulation Defects/Hematologic Disorders

$17,307(4.4)$

$2(3.2)$

ix most common reasons for hospitalization, $\mathrm{n}(\%)$

Sepsis

(8.1)

章 
Table 4

Summary of differential distance between closest non-integrated hospital and closest integrated hospital for each PAC type.

\begin{tabular}{lll}
\hline & SNF & HHA \\
\hline Differential distance to vertically integrated hospital in miles, mean (SD) & $14.6(30.4)$ & $11.2(29.9)$ \\
Differential distance to informally integrated hospital in miles, mean (SD) & $22.8(30.0)$ & $15.4(32.2)$ \\
\hline
\end{tabular}


Table 5

First stage results, regression of integration status on differential distance.

\begin{tabular}{llllll}
\hline & \multicolumn{2}{l}{$\begin{array}{l}\text { Based on distance to closest hospital vertically } \\
\text { integrated with PAC }\end{array}$} & & \multicolumn{2}{l}{$\begin{array}{l}\text { Based on distance to closest hospital informally } \\
\text { integrated with PAC }\end{array}$} \\
\cline { 2 - 3 } & SNF & HHA & SNF & HHA \\
\hline Differential distance & $-0.000781^{* * * *}$ & $-0.00276^{* * *}$ & $-0.00170^{* * * *}$ & $-0.00336^{* * * *}$ \\
& $(-11.25)$ & $(-14.69)$ & $(-20.71)$ & $(-22.54)$ \\
N of discharges & $2,651,748$ & $1,318,577$ & $2,651,748$ & $1,318,577$ \\
$\mathbf{N}$ of provider pairs & 87,440 & 56,093 & 87,440 & 56,093 \\
\hline
\end{tabular}

Note: Robust t-statistics in parentheses.

**** Signifies $\mathrm{p}<.01$ 


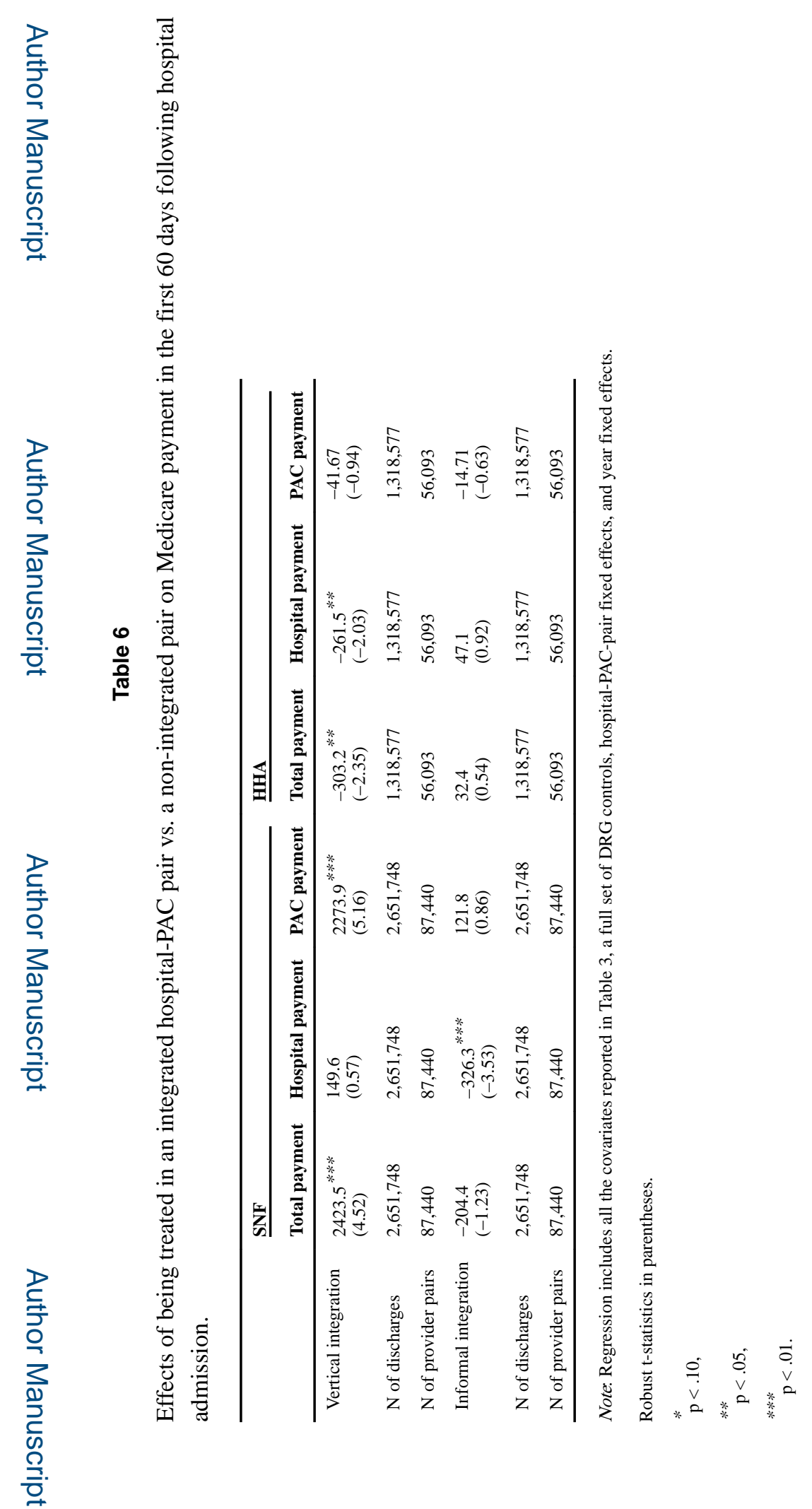

J Health Econ. Author manuscript; available in PMC 2018 September 21. 


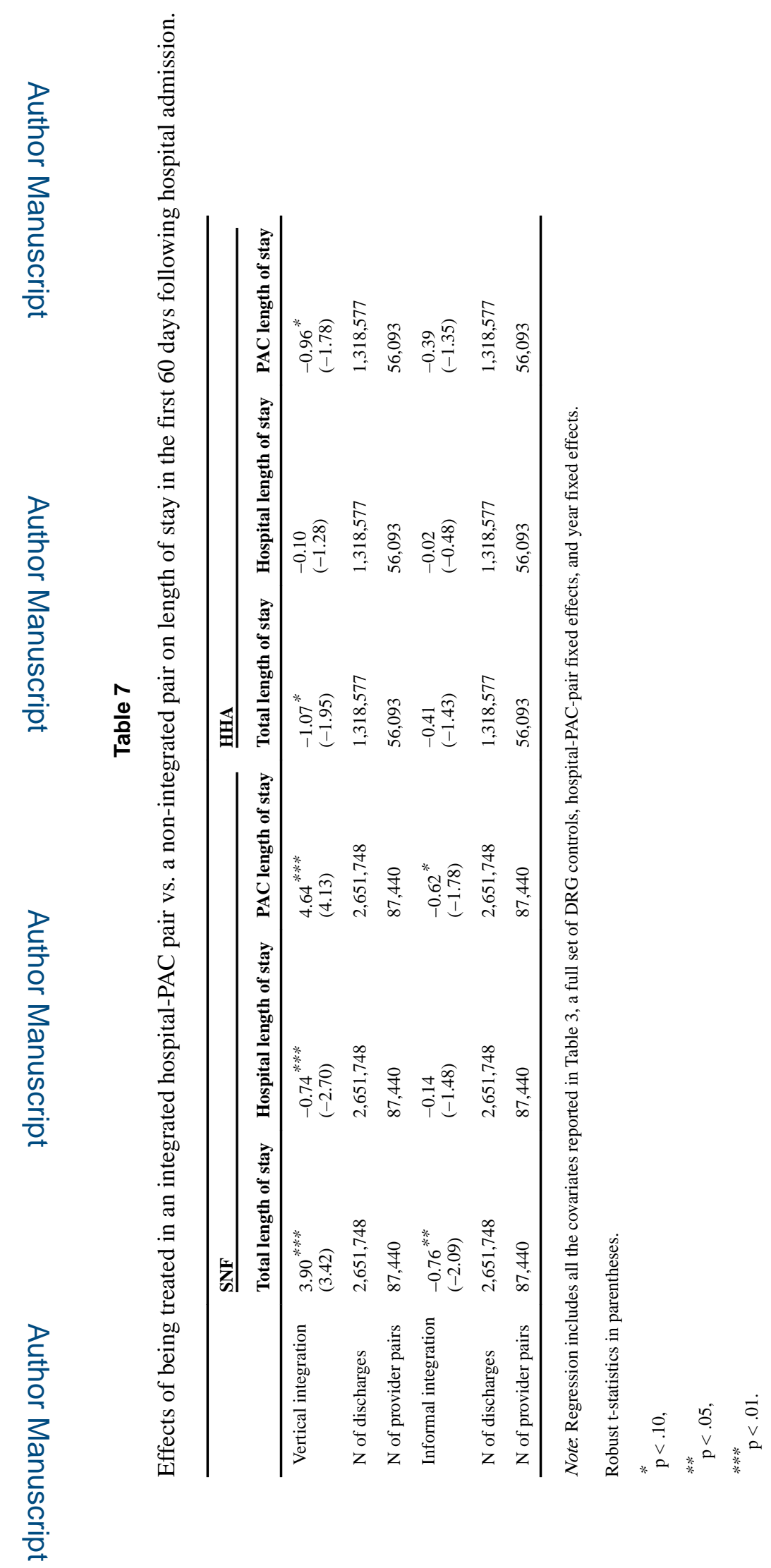

$J$ Health Econ. Author manuscript; available in PMC 2018 September 21. 


\section{Table 8}

Effects of being treated in an integrated hospital-PAC pair vs. a non-integrated pair on rehospitalization or death within 30 days of hospital discharge.

\begin{tabular}{lll}
\hline & \multicolumn{2}{l}{ Readmission } \\
\cline { 2 - 3 } & SNF & HHA \\
\hline Vertical integration & $\begin{array}{l}-0.054^{* * *} \\
(-1.96)\end{array}$ & $\begin{array}{l}-0.004 \\
(-0.49)\end{array}$ \\
N of discharges & $2,651,748$ & $1,318,577$ \\
N of provider pairs & 87,440 & 56,093 \\
Informal integration & 0.005 & -0.002 \\
& $(0.54)$ & $(-0.47)$ \\
N of discharges & $2,651,748$ & $1,318,577$ \\
$\mathbf{N}$ of provider pairs & 87,440 & 56,093 \\
\hline
\end{tabular}

Note: Regression includes all the covariates reported in Table 3, a full set of DRG controls, hospital-PAC-pair fixed effects, and year fixed effects.

Robust t-statistics in parentheses.

$\mathrm{p}<.10$,

* $\mathrm{p}<.05$

**** $\mathrm{p}<.01$. 


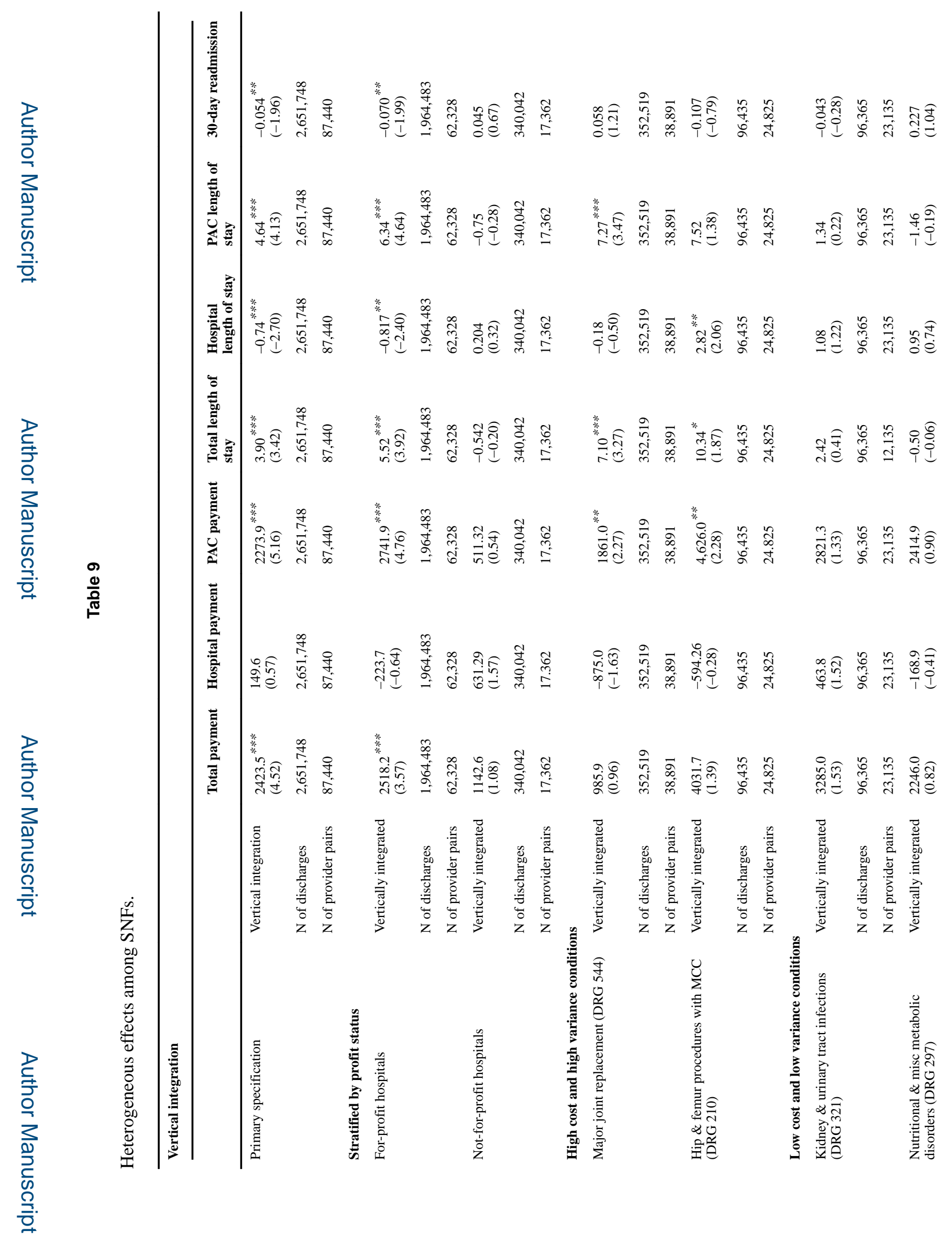

J Health Econ. Author manuscript; available in PMC 2018 September 21. 


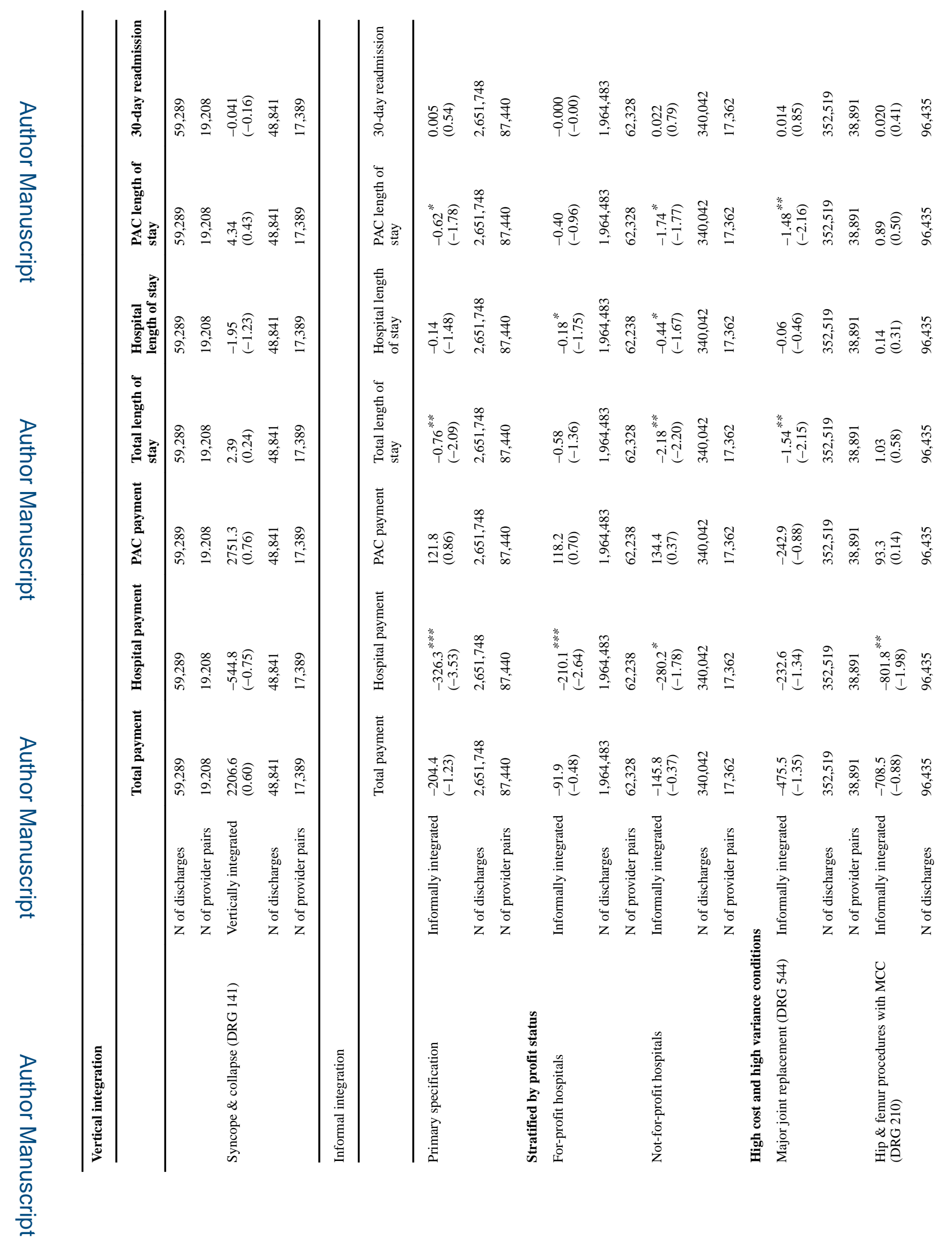

J Health Econ. Author manuscript; available in PMC 2018 September 21. 


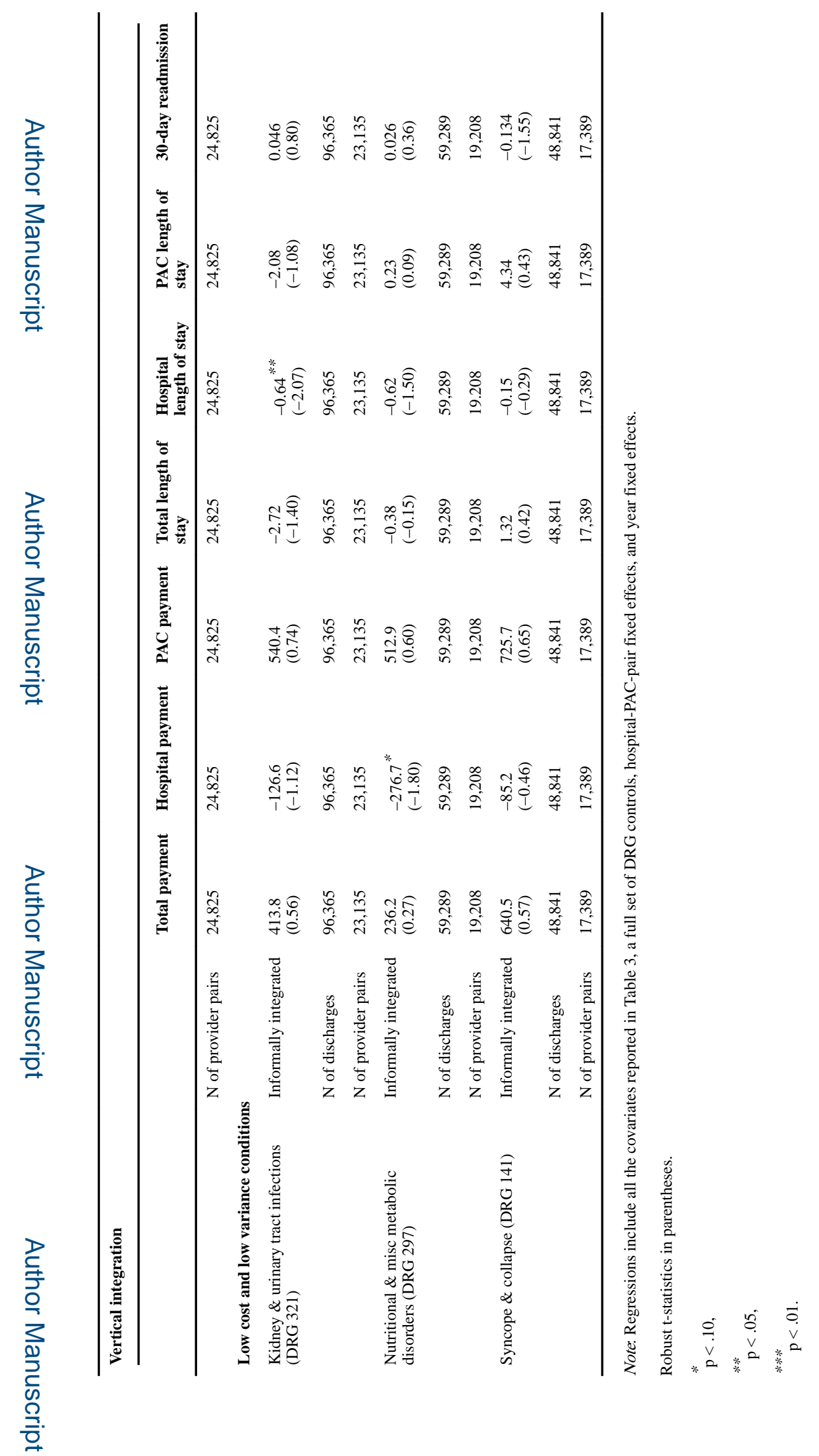

J Health Econ. Author manuscript; available in PMC 2018 September 21. 


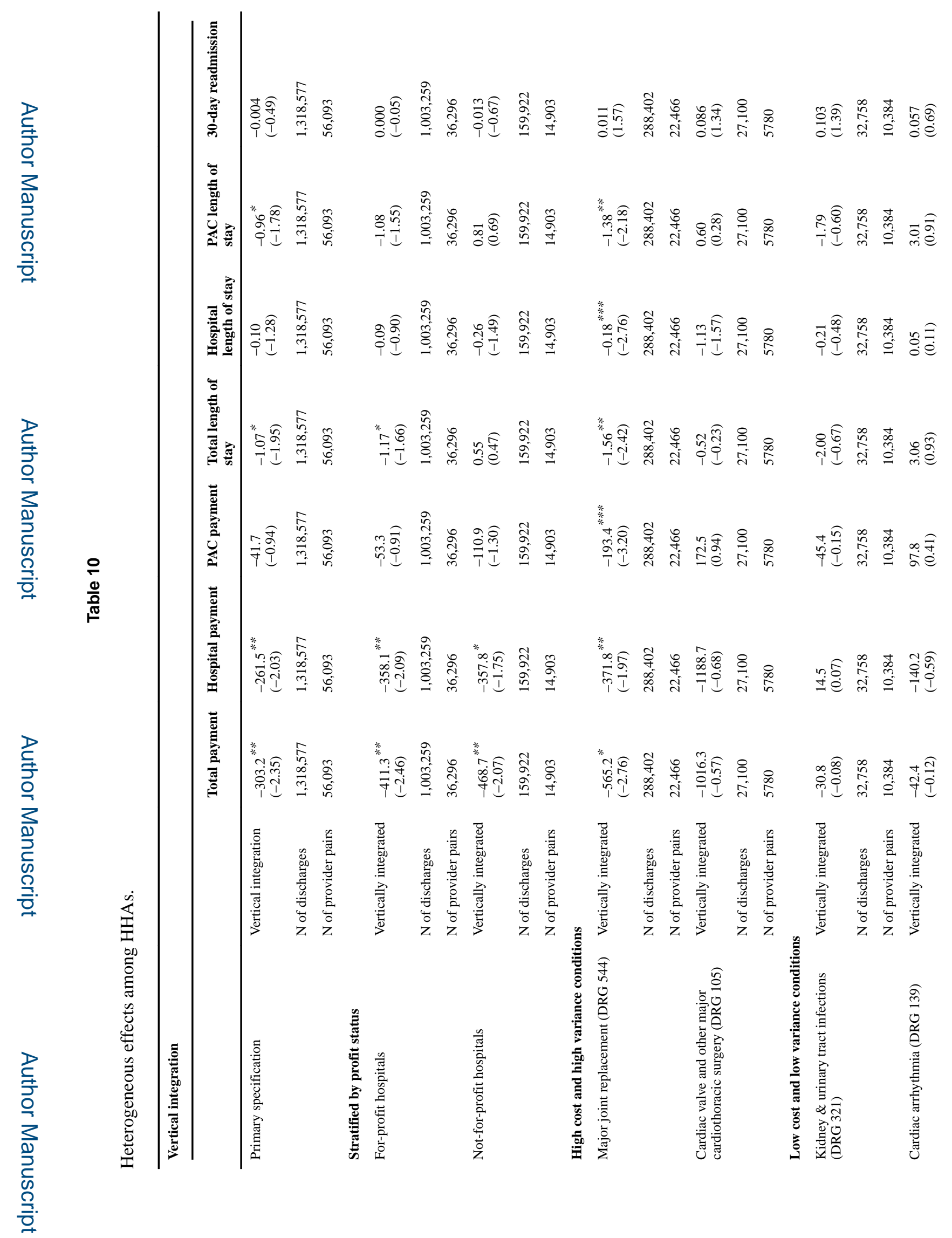

J Health Econ. Author manuscript; available in PMC 2018 September 21. 


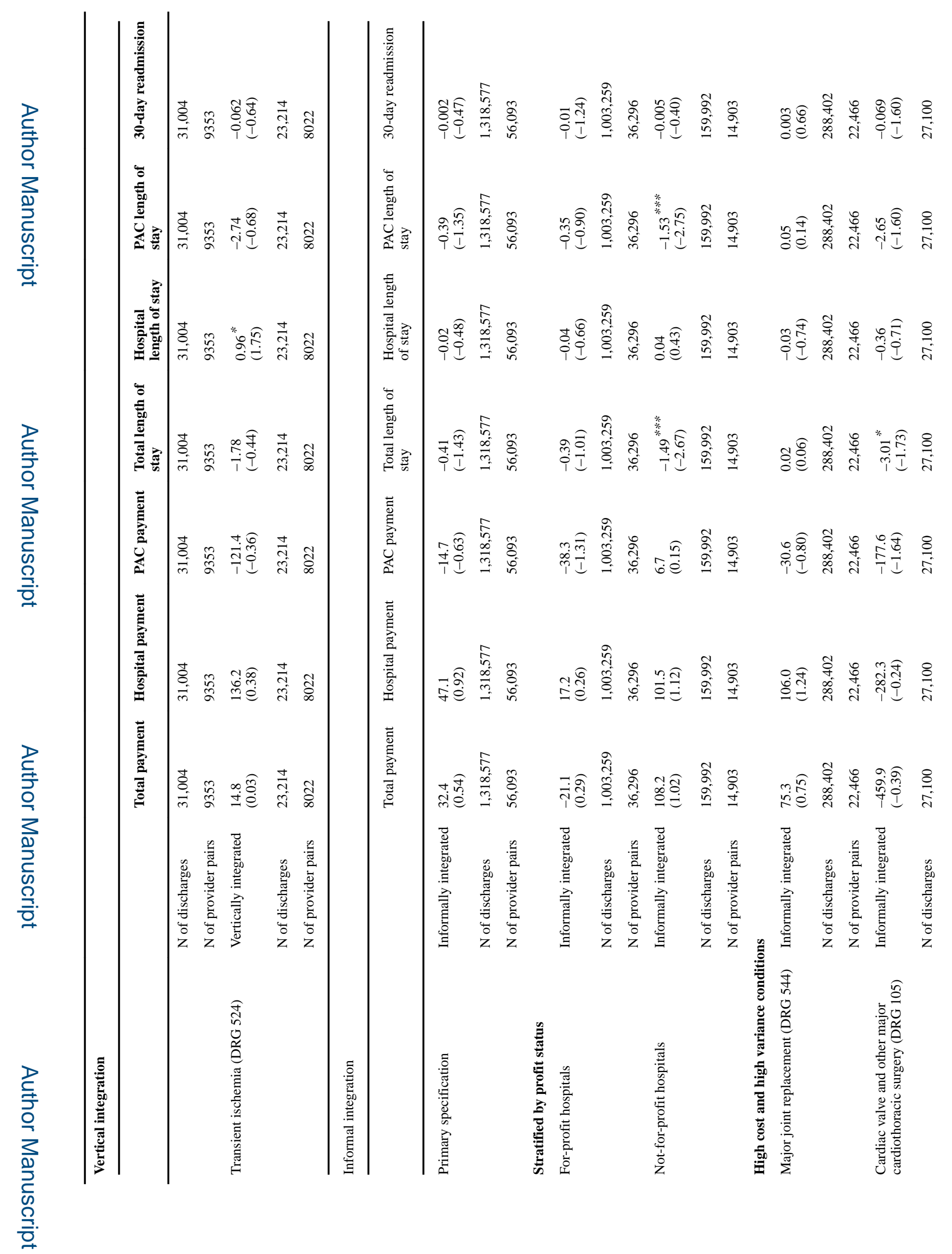

$J$ Health Econ. Author manuscript; available in PMC 2018 September 21. 


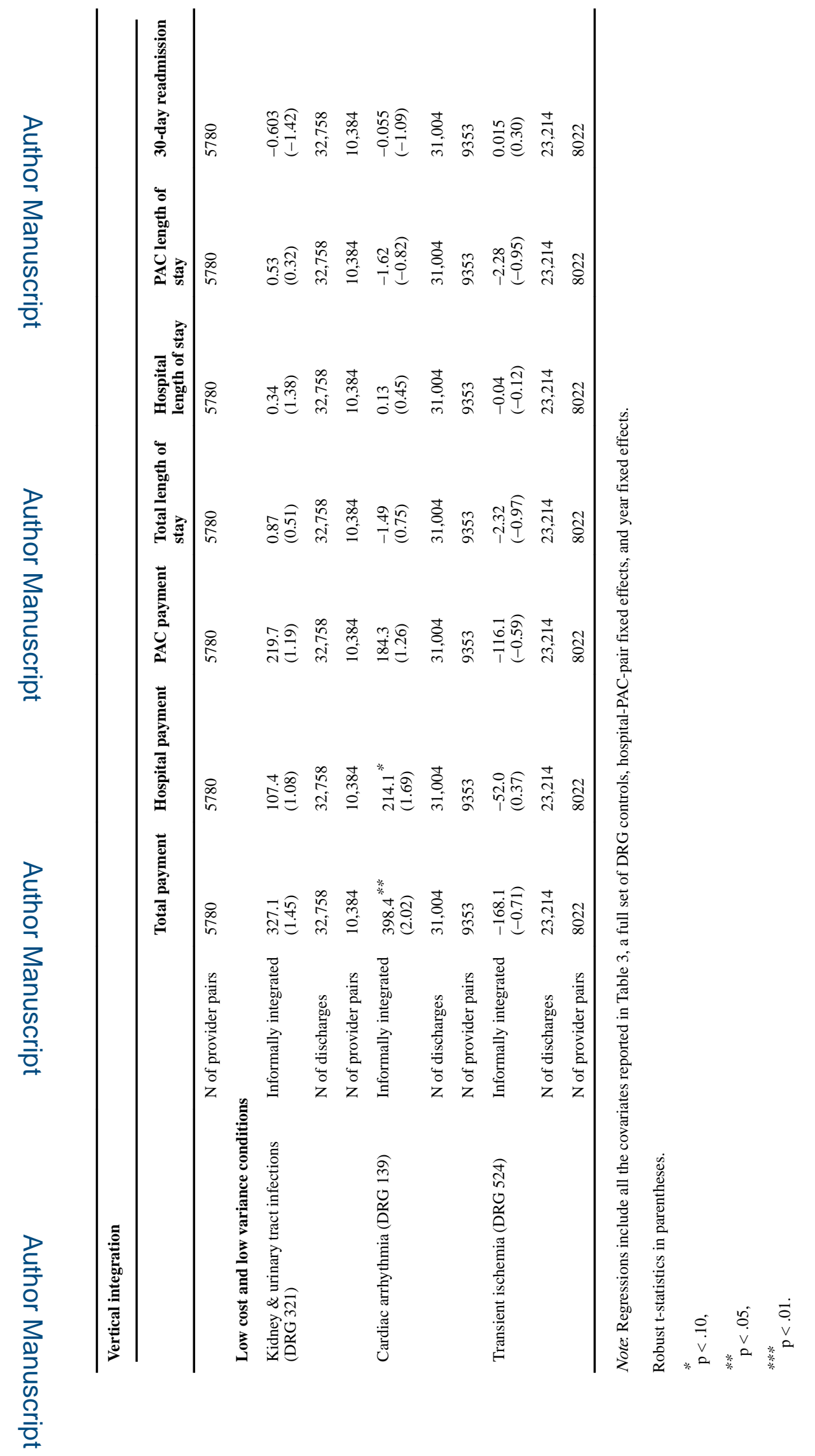

J Health Econ. Author manuscript; available in PMC 2018 September 21. 